\title{
Importance of molecular genetic analysis in the diagnosis and classification of congenital hypothyroidism
}

\author{
Héctor M. Targovnik
}

Received: 27 September 2013/ Accepted: 30 September 2013/Published online: 16 October 2013

(C) Springer Science+Business Media New York 2013

Congenital hypothyroidism $(\mathrm{CH})$ is the most frequent endocrine disease in infants, with the prevalence ratio in the range of 1:2,000-1:4,000 newborns. It is characterized by elevated levels of TSH resulting from reduced thyroid function [1]. It is also one of the most common preventable causes of cognitive and motor deficits. Prevention of $\mathrm{CH}$ is based on carrier identification, genetic counseling, and prenatal diagnosis. Early detection and treatment of such infants, as a consequence of the neonatal-screening programs, are critical to normal brain development and physical growth. In neonates, a complete diagnosis of $\mathrm{CH}$ should include clinical examination, biochemical thyroid tests, ultrasound, and radioiodine or technetium scintigraphy. The article by Cangul et al. [2] in this issue of Endocrine provides additional information about the clinical and genetic analyses from four new patients with $\mathrm{CH}$, belonging to two unrelated consanguineous Turkish families, homozygous for nonsense mutations in the TG gene.

In the last two decades, significant progress has been made in understanding the pathophysiology of $\mathrm{CH}$. Genetic abnormalities in any of the key thyroid proteins can produce $\mathrm{CH}[3,4]$. Based on genetic alterations, the $\mathrm{CH}$ can be classified into two main groups: (i) those caused by disorders of thyroid gland development (dysembriogenesis or dysgenesis), which accounts for $80-85 \%$ of cases; and (ii)

H. M. Targovnik ( $\square)$

Laboratorio de Genética y Biología Molecular, Instituto de Inmunología, Genética y Metabolismo (INIGEM, CONICETUBA), Hospital de Clínicas "José de San Martín”, Av. Córdoba 2351, Cuarto Piso, Sala 5, C1120AAR Buenos Aires, Argentina e-mail: htargovnik@conicet.gov.ar

H. M. Targovnik

Cátedra de Genética y Biología Molecular (FFyB-UBA),

Junín 954, C1113AAD Buenos Aires, Argentina by defects in any step of thyroid hormone synthesis (dyshormonogenesis), which accounts for remaining 15-20\% of cases [3, 4]. Thyroid dysembryogenesis results from a thyroid gland that is completely absent in orthotopic or ectopic location (agenesis or athyreosis), severely reduced in size but in the proper position in the neck (orthotopic hypoplasia) or located in an unusual position (thyroid ectopy) at the base of the tongue or along the thyroglossal tract [4]. In a very minority of these patients $(5 \%)$, the $\mathrm{CH}$ is associated with mutations in genes responsible for the development or growth of thyroid follicular cells: NKX2.1 FOXE1, PAX-8, NKX2.5, and TSHR genes [3, 4]. Consequently, the molecular mechanisms underlying the defect in thyroid organogenesis in the great majority of cases remain to be elucidated. In contrast, thyroid dyshormonogenesis has been linked in more than $90 \%$ of cases to mutations in the sodium iodide symporter (NIS), SLC26A4 (which encodes pendrin), thyroperoxidase (TPO), dual oxidase 2 (DUOX2), DUOX maturation factor 2 (DUOXA2), dehalogenase 1 (DEHAL1), and thyroglobulin (TG) genes [3, 4]. These mutations produce a heterogeneous spectrum of congenital goitrous hypothyroidism, usually transmitted in an autosomal recessive mode.

Dyshormonogenesis due to TG gene mutations is a rare cause of $\mathrm{CH}$ with an estimated incidence of approximately 1 in 100,000 newborns [3]. The clinical spectrum ranges from euthyroid to mild or severe hypothyroidism. The majority of patients have congenital goiter or goiter appearing shortly after birth [3]. To date, 70 deleterious mutations in the human TG gene have been identified and characterized: 15 splice site mutations, 14 nonsense mutations, 28 missense mutations, 10 deletions (eight single and two involving a large number of nucleotides), and three single nucleotide insertions [3-5]. Recently, a novel large imperfect DNA inversion within the TG gene was identified by the strategy 
of I-PCR [6]. This aberration was not detectable by normal sequencing of the exons and exon/intron boundaries.

Cangul et al. [2] describe the first report in which inactivating mutations in exon 9 was identified in the human TG gene and detected two new nonsense TG mutations: p.Q630X and p.W637X. Previously, a p.R697X mutation in the exon 9 of TG gene has been described in Afrikander cattle [7]. This mutation is thus removed from the transcripts by exon 9 skipping, and there is a preferential accumulation in the goiter of a shorter mRNA [7]. This compensatory mechanism originated from an alternative splicing in the cattle, unidentified until now in humans. It is interesting to note that the mRNA mutants could represent adequate targets for nonsense-mediated mRNA decay (NMD) pathway, a mechanism that degrades selectively mRNAs that contain premature stop codons. It is well documented also that truncated TG devoid of the ACHE-like domain may cause TG retention in the ER and premature degradation [8]. In addition to NMD pathway and ER storage diseases as pathophysiological mechanisms in the generation of $\mathrm{CH}$, these truncated mutants have impairment in thyroid hormone synthesis. To complete this scenario, a second and remarkably finding was observed by Cangul et al. [2], the two affected siblings with p.W637X mutation in the TG gene showed surprisingly thyroid hypoplasia. As mentioned above, the patients with TG deficiency have different degrees of goiter due to proliferative effect of the high and constant levels of TSH. However, mutations in the TSHR are the most frequent genetic cause of hypoplasia, event excluded by the authors in this article by linkage analysis. In agreement with this finding but in contrast to published human patients and other animal models of $\mathrm{CH}$, the $\mathrm{rdw} / \mathrm{rdw}$ rat (derived from the Wistar-Imamichi strain) presented also a hypoplastic thyroid gland associated with TG mutation, despite elevated circulating levels of TSH and reduced serum T3 and T4 [9]. The corresponding mutation in the rdw TG was a single nucleotide change, c.6958G $>C$ that generates an amino acid substitution in the ACHE-like domain (p.G2300R) and impairment of intracellular transport [9]. One hypothesis to explain the hypoplasia is that in $\mathrm{rdw} / \mathrm{rdw}$ rats, the interactions between TGp.G2300R and Erp72 chaperone predispose to thyrocyte cell death, consequently preventing compensatory goiter [10]. The reason for this phenomenon in the present article remains to be elucidated. From a practical perspective and according to these findings, it is necessary to integrate the investigation of mutations in the TG gene into genetic analyses of cases of hypoplasia not associated with mutations in the TSHR gene or in genes responsible for thyroid development.

Many questions remain to be answered about the genotype and phenotype $\mathrm{CH}$. Molecular diagnosis has been traditionally established by PCR-based approaches followed by systematic sequence analysis. In the phenotype caused by a monoallelic defect, it is likely that the apparent absence of a second mutation could be explained by technical limitations of the direct sequencing analysis. In the near future, the implementation of new techniques revolutionizing our understanding of the genetic pathophysiology of the $\mathrm{CH}$, including the use of gene chips, multiplex ligationdependent probe amplification and next generationsequencing technology will allow us to identify rapidly mutations in remote regions and to characterize large deletions, duplications, or inversions.

Acknowledgments H. M. Targovnik is an established investigator of the Consejo Nacional de Investigaciones Científicas y Técnicas (CONICET). This study was supported by Grants from the Universidad de Buenos Aires (20020100100594/2011): CONICET (PIP 2012/112-201101-00091), and FONCyT-ANPCyT-MINCyT (PICT 2010/05-1130 and PICT 2012/05-1090).

Conflict of interest Author declares that they have no conflict of interest.

\section{References}

1. M.V. Rastogi, S.H. LaFranchi, Congenital hypothyroidism. Orphanet J. Rare Dis. 5, 17 (2010)

2. H. Cangul, K. Boelaert, M. Dogan et al., Novel truncating thyroglobulin gene mutations associated with congenital hypothyroidism. Endocrine (2013). doi:10.1007/s12020-013-0027-7

3. H.M. Targovnik, S.A. Esperante, C.M. Rivolta, Genetics and phenomics of hypothyroidism and goiter due to thyroglobulin mutations. Mol. Cell. Endocrinol. 322, 44-55 (2010)

4. H.M. Targovnik, C.E. Citterio, C.M. Rivolta, Thyroglobulin Gene Mutations in Congenital Hypothyroidism. Horm. Res. Paediatr. 75, 311-321 (2011)

5. H.M. Targovnik, Thyroglobulin structure, function and biosynthesis, in Werner and Ingbar's the thyroid: a fundamental and clinical text, 10th edn., ed. by L. Braverman, D. Cooper (Lippincott Williams \& Wilkins, Philadelphia, 2012), pp. 74-92

6. C.E. Citterio, L.C. Rossetti, P.F. Souchon et al., Novel mutational mechanism in the thyroglobulin gene: imperfect DNA inversion as a cause for hereditary hypothyroidism. Mol. Cell. Endocrinol. 381, 220-229 (2013)

7. M.H. Ricketts, M.J. Simons, J. Parma et al., A nonsense mutation causes hereditary goitre in the Afrikander cattle and unmasks alternative splicing of thyroglobulin transcripts. Proc. Natl. Acad. Sci. USA 84, 3181-3184 (1987)

8. J. Lee, B. Di Jeso, P. Arvan, The cholinesterase-like domain of thyroglobulin functions as an intramolecular chaperone. J. Clin. Invest. 118, 2950-2958 (2008)

9. P.S. Kim, M. Ding, S. Menon et al., A missense mutation G2320R in the thyroglobulin gene causes non-goitrous congenital primary hypothyroidism in the WIC-rdw rat. Mol. Endocrinol. 14, 1944-1953 (2000)

10. S. Menon, J. Lee, W.A. Abplanalp, Oxidoreductase interactions include a role for ERp72 engagement with mutant thyroglobulin from the rdw/rdw rat dwarf. J. Biol. Chem. 282, 6183-6191 (2007) 